CRYSTALLOGRAPHIC COMMUNICATIONS

ISSN 2056-9890

Received 29 November 2015

Accepted 2 December 2015

Edited by W. T. A. Harrison, University of Aberdeen, Scotland

Keywords: crystal structure; supramolecular structure; hydrogen bonds; halogen-pyridine interactions

CCDC reference: 1440028

Supporting information: this article has

supporting information at journals.iucr.org/e

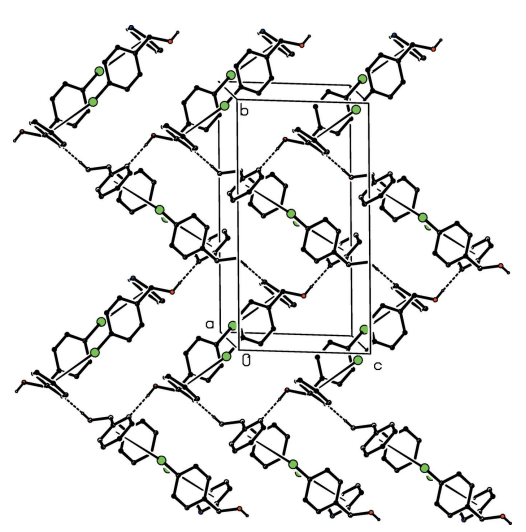

OPEN $\odot$ ACCESS

\section{Crystal structure of (RS)-(4-chlorophenyl)(pyridin- 2-yl)methanol}

\author{
Badiadka Narayana, ${ }^{\text {a } B a l l a d k a ~ K . ~ S a r o j i n i, ~}{ }^{\mathrm{b}}$ Hemmige S. Yathirajan, ${ }^{\mathrm{c} *}$ Ravindranath \\ Rathore $^{\mathrm{d}}$ and Christopher Glidewell ${ }^{\mathrm{e}}$
}

${ }^{\mathbf{a}}$ Department of Studies in Chemistry, Mangalore University, Mangalagangothri 574 199, India, ${ }^{\mathbf{b}}$ Department of Studies in Industrial Chemistry, Mangalore University, Mangalagangothri 574 199, India, 'Department of Studies in Chemistry, University of Mysore, Manasagangotri, Mysuru 570 006, India, dDepartment of Biotechnology, Dayananda Sagar College of Engineering, Bengaluru 560 078, India, and ${ }^{\mathbf{e}}$ School of Chemistry, University of St Andrews, Fife KY16 9ST, Scotland. *Correspondence e-mail: yathirajan@hotmail.com

In the title racemic compound, $\mathrm{C}_{12} \mathrm{H}_{10} \mathrm{ClNO}$, the dihedral angle between the benzene and pyridine rings is $74.34(6)^{\circ}$. In the crystal, the molecules are linked by $\mathrm{O}-\mathrm{H} \cdots \mathrm{N}$ hydrogen bonds, forming zigzag $C(5)$ [001] chains in which alternating $R$ - and $S$-configuration molecules are related by $c$-glide symmetry. In addition, inversion-related pairs of molecules are linked into dimers by pairs of weak $\mathrm{C}-\mathrm{Cl} \cdots \pi$ (pyridyl) interactions, which link the hydrogen-bonded chains into (100) sheets. Structural comparisons are drawn with a number of related compounds.

\section{Chemical context}

Simply substituted diphenylmethanols, $R \mathrm{Ph}_{2} \mathrm{COH}$, exhibit a very rich diversity of supramolecular arrangements, including isolated molecules, hydrogen-bonded dimers, trimers, tetramers and hexamers, as well as continuous hydrogen-bonded chains (Ferguson et al., 1992, 1994, 1995). The predominant mode of molecular association in these structures involves $\mathrm{O}-\mathrm{H} \cdots \mathrm{O}$ hydrogen bonds, although $\mathrm{O}-\mathrm{H} \cdots \pi$ (arene) interactions are sometimes present. It is therefore of considerable interest to investigate the influence of an addition potential acceptor of hydrogen bonds as achieved, for example, by the replacement of one of the phenyl rings by an isosteric pyridyl substituent. Here we report the molecular and supramolecular structure of ( $R S)$-4-chlorophenyl(pyridin-2yl)methanol (I) (Fig. 1), which shows some striking structural differences from the simpler, non-chlorinated analogue phenyl(pyridin-2-yl)methanol, whose structure has been reported recently (Kim \& Kang, 2014; Tsang et al., 2015).<smiles>OC(c1ccc(Cl)cc1)c1ccccn1</smiles>

\section{Structural commentary}

The molecules of compound (I) contain a stereogenic centre at atom $\mathrm{C} 1$ (Fig. 1) and the reference molecule was selected as one having the $R$-configuration at atom $\mathrm{C} 1$. The centrosym- 
Table 1

Selected torsion angles $\left({ }^{\circ}\right)$.

\begin{tabular}{llll}
\hline $\mathrm{O} 1-\mathrm{C} 1-\mathrm{C} 11-\mathrm{C} 12$ & $-51.14(17)$ & $\mathrm{C} 11-\mathrm{C} 1-\mathrm{O} 1-\mathrm{H} 1 A$ & $-180.0(17)$
\end{tabular}

$\mathrm{O} 1-\mathrm{C} 1-\mathrm{C} 22-\mathrm{N} 21-156.41(13)$

metric space group confirms that compound (I) has crystallized as a racemic mixture.

Both of the rings are rotated out of the plane of the central C11-C1-C22 fragment, which makes dihedral angles of 70.69 (2) and $84.66(9)^{\circ}$ with the phenyl and pyridyl rings, respectively. The dihedral angle between the rings is $74.34(6)^{\circ}$, and this value is very similar to the value of $71.42(10)^{\circ}$ reported (Kim \& Kang, 2014) for the corresponding angle in the non-chlorinated analogue, compound (II). The general conformational similarity between the molecules of compounds (I) and (II) is shown by the torsional angles $\mathrm{O}-\mathrm{C}-\mathrm{C}-\mathrm{C}$ and $\mathrm{O}-\mathrm{C}-\mathrm{C}-\mathrm{N}$ (Table 1 ), where the corresponding angles for the $R$-enantiomer of (II) [the reference molecule was actually selected (Kim \& Kang, 2014) as one having the $S$-configuration] are $49.0(4)$ and $-150.6(2)^{\circ}$, respectively.

However, one point of difference between the conformations in compounds (I) and (II) centres on the locations of the hydroxyl $\mathrm{H}$ atoms. In compound (I), this atom is antiperiplanar to atom C11 (Table 1), but the corresponding torsional angle for the $R$-enantiomer of (II) is $-67(2)^{\circ}$. This difference in hydroxyl group conformations is probably associated with the different patterns of hydrogen-bonded supramolecular aggregation in compounds (I) and (II), as discussed below.

\section{Supramolecular interactions}

The molecules of compound (I) are linked by $\mathrm{O}-\mathrm{H} \cdots \mathrm{N}$ hydrogen bonds (Table 2), forming zigzag $C(5)$ chains running parallel to the [001] direction. The chain containing the reference molecule at $(x, y, z)$ consists of molecules which are related by the $c$-glide plane at $y=\frac{1}{4}$, so that molecules of $R$ configuration and $S$-configuration alternate along the chain

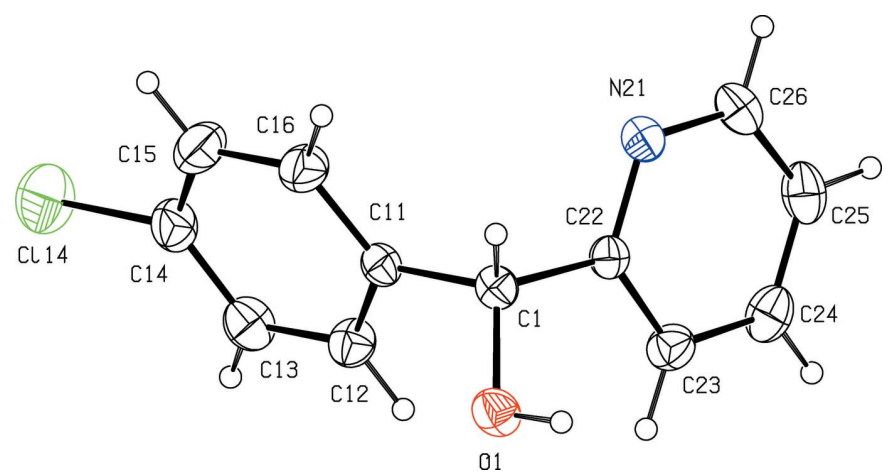

Figure 1

The molecular structure of the $R$-enantiomer of compound (I), showing the atom-labelling scheme. Displacement ellipsoids are drawn at the $30 \%$ probability level.
Table 2

Hydrogen-bond geometry $\left(\AA{ }^{\circ}\right)$.

\begin{tabular}{lllll}
\hline$D-\mathrm{H} \cdots A$ & $D-\mathrm{H}$ & $\mathrm{H} \cdots A$ & $D \cdots A$ & $D-\mathrm{H} \cdots A$ \\
\hline $\mathrm{O} 1-\mathrm{H} 1 A \cdots \mathrm{N} 21^{\mathrm{i}}$ & $0.84(2)$ & $2.01(2)$ & $2.8444(18)$ & $176(2)$ \\
\hline
\end{tabular}

Symmetry code: (i) $x,-y+\frac{1}{2}, z+\frac{1}{2}$.

(Fig. 2). Two chains of this type, related to one another by inversion, pass through each unit cell.

The crystal structure of compound (I) contains neither C$\mathrm{H} \cdots \pi$ hydrogen bonds nor $\pi-\pi$ stacking interactions. There is, however, a single short $\mathrm{C}-\mathrm{Cl} \cdots \pi$ contact with geometric parameters $\mathrm{Cl} \cdots C g^{\mathrm{i}}=3.5280(10) \AA, C \cdots C g^{\mathrm{i}}=5.1785$ (19) $\AA$ and $\mathrm{C}-\mathrm{Cl} \cdots \mathrm{Cg}^{\mathrm{i}}=157.79(7)^{\circ}$ [symmetry code: (i) $1-x,-y$, $-z$ ] where $C g$ represents the centroid of the pyridine ring. This $\mathrm{Cl} \cdots \mathrm{Cg}$ distance is slightly shorter than the average distance, $3.6 \AA$, deduced (Imai et al., 2008) from database analysis in a study which concluded that such interactions were attractive, with interaction energies of $c a 2 \mathrm{kcal} \mathrm{mol}^{-1}$, comparable to those typical of weak hydrogen bonds

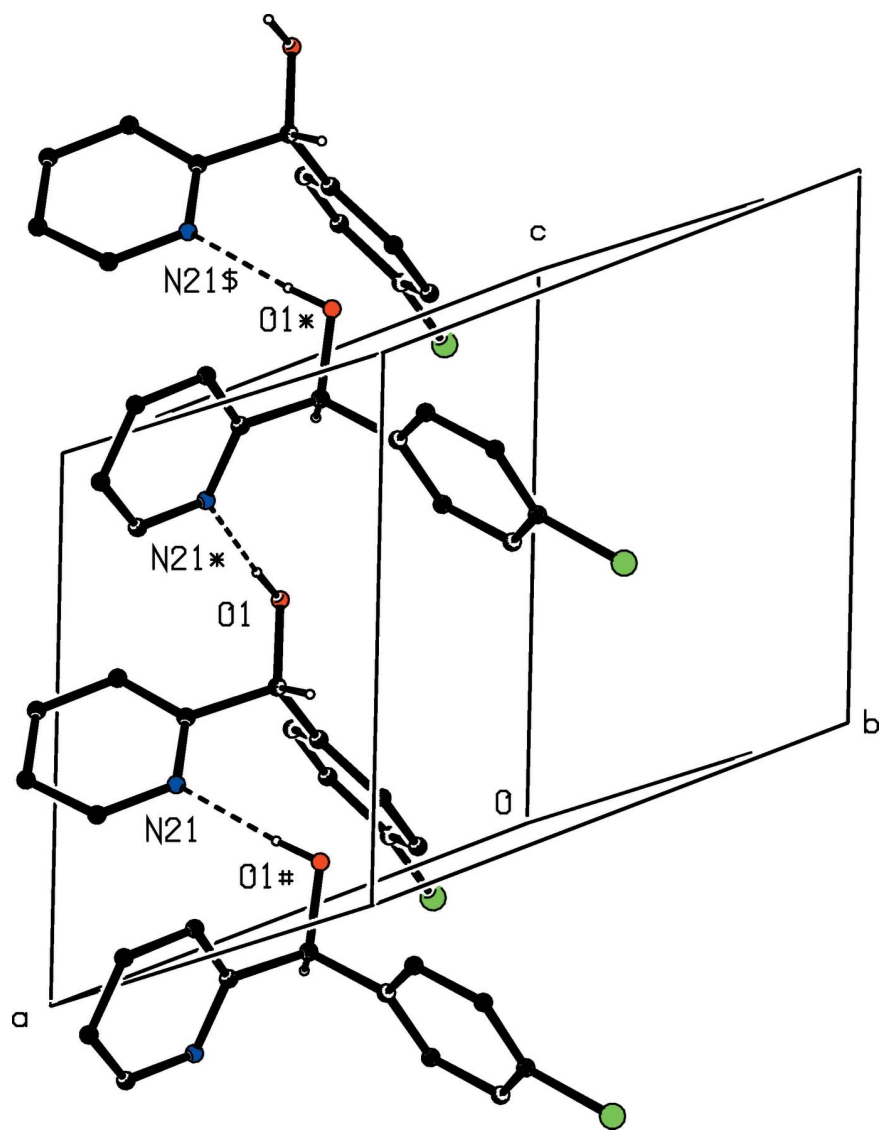

Figure 2

Part of the crystal structure of compound (I), showing the formation of a hydrogen-bonded $C(5)$ chain containing alternating enantiomers and running parallel to [001]. For the sake of clarity, the $\mathrm{H}$ atoms bonded to the ring $\mathrm{C}$ atoms have been omitted. The atoms marked with an asterisk $(*)$, a hash (\#) or a dollar sign (\$) are at the symmetry positions $\left(x, \frac{1}{2}-y\right.$, $\left.\frac{1}{2}+z\right),\left(x, \frac{1}{2}-y,-\frac{1}{2}+z\right)$ and $(x, y, 1+z)$, respectively. 


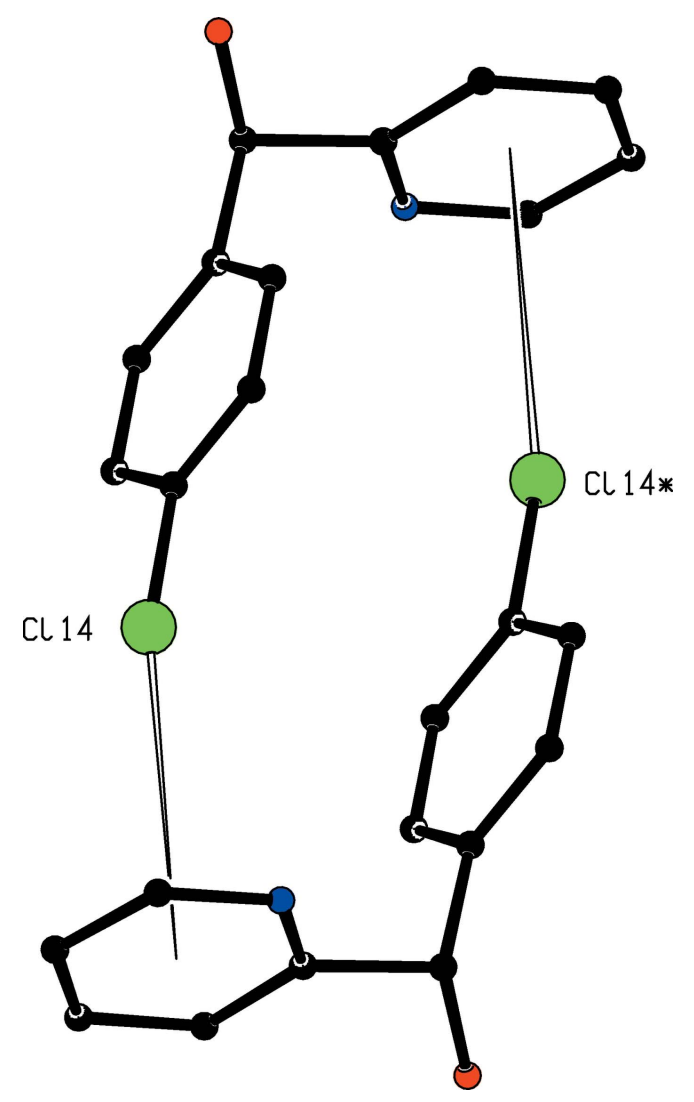

Figure 3

A centrosymmetric dimer in in the crystal of (I) in which the molecules are linked by $\mathrm{C}-\mathrm{Cl} \cdots \pi$ interactions, shown as hollow lines. For the sake of clarity, all of the $\mathrm{H}$ atoms have been omitted. The $\mathrm{Cl}$ atom marked with an asterisk $(*)$ is at the symmetry position $(1-x,-y,-z)$.

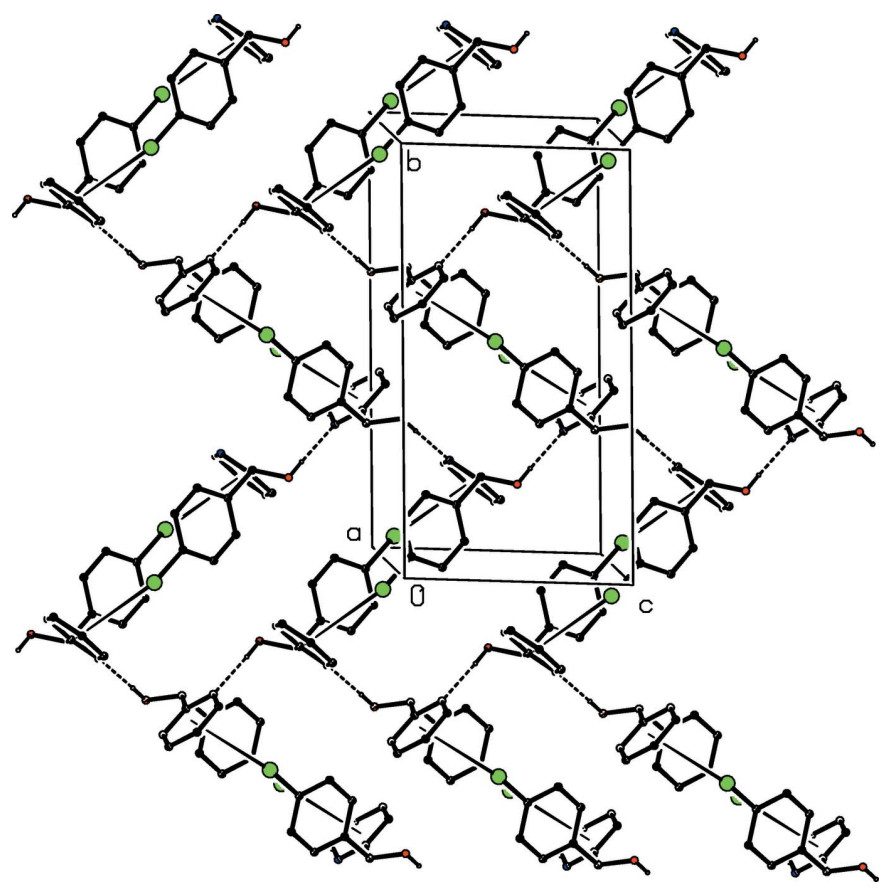

Figure 4

A view of part of the crystal structure of (I), showing the formation of a sheet parallel to (001) built from hydrogen-bonded chains linked by $\mathrm{C}-$ $\mathrm{Cl} \cdots \pi$ interactions. For the sake of clarity, the $\mathrm{H}$ atoms bonded to $\mathrm{C}$ atoms have all been omitted.<smiles>OC(c1ccc(Cl)cc1)c1ccccn1</smiles>

(I)<smiles>OC(c1ccccc1)c1ccncc1</smiles>

(VI)<smiles>[R]C(O)c1ccccn1</smiles><smiles>OC(c1ccccc1)c1ccccn1</smiles>

(II)
(III) $R=1,2-\mathrm{C}_{2} \mathrm{~B}_{10} \mathrm{H}_{11}$

(IV) $R=1,7-\mathrm{C}_{2} \mathrm{~B}_{10} \mathrm{H}_{11}$

(V) $R=1,12-\mathrm{C}_{2} \mathrm{~B}_{10} \mathrm{H}_{11}$<smiles>OC(c1ccccc1)c1ccccc1</smiles>

(VII)<smiles>OC(c1c(F)c(F)c(F)c(F)c1F)c1c(F)c(F)c(F)c(F)c1F</smiles>

(VIII)

Figure 5

Related compounds.

(Desiraju \& Steiner, 1999). In compound (I), this interaction links inversion-related pairs of molecules into cyclic centrosymmetric dimers (Fig. 3).

The overall effect of the $\mathrm{C}-\mathrm{Cl} \cdots \pi$ interaction in (I) is to link the hydrogen-bonded chain containing molecules related by the $c$-glide plane at $y=\frac{1}{4}$ directly to the two chains that contain molecules related by the glide planes at $y=-\frac{1}{4}$ and $y=\frac{3}{4}$, respectively, and propagation by translation of this interaction links the hydrogen-bonded chains along [001] into a sheet lying parallel to (100) (Fig. 4), but there are no direction-specific interactions between adjacent sheets.

\section{Structural comparisons with related compounds}

It is of interest briefly to compare the supramolecular assembly in compound (I), mediated by $\mathrm{O}-\mathrm{H} \cdots \mathrm{N}$ hydrogen bonds and $\mathrm{C}-\mathrm{Cl} \cdots \pi$ interactions, with the assembly in some closely related compounds (II)-(VIII) (see Fig. 5), and particularly with compound (II), whose constitution differs from that of (I) only in lacking the chloro substituent.

The molecules of compound (II) are linked into $C(5)$ chains by $\mathrm{O}-\mathrm{H} \cdots \mathrm{N}$ hydrogen bonds (Kim \& Kang, 2014; Tsang et al., 2015), as in compound (I), but in (II) helical chains are 
Table 3

Experimental details.

\begin{tabular}{|c|c|}
\hline \multicolumn{2}{|l|}{ Crystal data } \\
\hline Chemical formula & $\mathrm{C}_{12} \mathrm{H}_{10} \mathrm{ClNO}$ \\
\hline$M_{\mathrm{r}}$ & 219.66 \\
\hline Crystal system, space group & Monoclinic, $P 2_{1} / c$ \\
\hline Temperature $(\mathrm{K})$ & 295 \\
\hline$a, b, c(\AA)$ & $8.4309(6), 16.1488(11), 8.6878(6)$ \\
\hline$\beta\left(^{\circ}\right)$ & $112.994(2)$ \\
\hline$V\left(\AA^{3}\right)$ & $1088.85(13)$ \\
\hline$Z$ & 4 \\
\hline Radiation type & Мо $K \alpha$ \\
\hline$\mu\left(\mathrm{mm}^{-1}\right)$ & 0.32 \\
\hline Crystal size $(\mathrm{mm})$ & $0.40 \times 0.30 \times 0.20$ \\
\hline \multicolumn{2}{|l|}{ Data collection } \\
\hline Diffractometer & Bruker APEXII CCD \\
\hline Absorption correction & $\begin{array}{l}\text { Multi-scan (SADABS; Sheldrick, } \\
\text { 2003) }\end{array}$ \\
\hline$T_{\min }, T_{\max }$ & $0.719,0.938$ \\
\hline $\begin{array}{l}\text { No. of measured, independent and } \\
\text { observed }[I>2 \sigma(I)] \text { reflections }\end{array}$ & $11481,2510,1860$ \\
\hline$R_{\text {int }}$ & 0.030 \\
\hline$(\sin \theta / \lambda)_{\max }\left(\AA^{-1}\right)$ & 0.651 \\
\hline \multicolumn{2}{|l|}{ Refinement } \\
\hline$R\left[F^{2}>2 \sigma\left(F^{2}\right)\right], w R\left(F^{2}\right), S$ & $0.045,0.118,1.06$ \\
\hline No. of reflections & 2510 \\
\hline No. of parameters & 139 \\
\hline $\mathrm{H}$-atom treatment & $\begin{array}{l}\mathrm{H} \text { atoms treated by a mixture of } \\
\text { independent and constrained } \\
\text { refinement }\end{array}$ \\
\hline$\Delta \rho_{\max }, \Delta \rho_{\min }\left(\mathrm{e} \AA^{-3}\right)$ & $0.21,-0.39$ \\
\hline
\end{tabular}

Computer programs: APEX2 and SAINT-Plus (Bruker, 2012), SHELXS97 (Sheldrick, 2008), SHELXL2014 (Sheldrick, 2015) and PLATON (Spek, 2009).

built from molecules related by $2_{1}$ screw axes in space group $P n a 2_{1}$, whereas in (I) zigzag chains are built from molecules related by glide planes. Hence in compound (II) each chain is homochiral, with equal numbers of chains built only from molecules having the $R$-configuration or only from molecules having the $S$-configuration: in (I), by contrast, each chain contains an alternation of the two enantiomers ( $c f$. Fig. 2).

Similar homochiral $C(5)$ chains are formed in each of the three isomeric carborane derivatives (III)-(V) (Tsang et al., 2015), regardless of whether they are crystallized as single enantiomers or as racemates. The structure of compound (VI), which is isomeric with (II) has been reported briefly (Shimada et al., 2003) but, unfortunately, no atomic coordinates have been deposited in the Cambridge Structural Database (Groom \& Allen, 2014). The structure report on (VI) concerns enantiomerically pure forms, in space group $P 2_{1} 2_{1} 2_{1}$, so that the formation of homochiral helical chains of $C(7)$ type, seems plausible.

Compound (VII), which differs from (I) and (II) in containing two unsubstituted phenyl rings but no pyridyl ring, crystallizes with $Z^{\prime}=2$ in space group $P 22_{1} 2_{1}$ (Ferguson et al., $1995)$ and the molecules are linked by $\mathrm{O}-\mathrm{H} \cdots \mathrm{O}$ hydrogen bonds to form $C_{2}^{2}(4)$ chains, but with no direction-specific interactions between adjacent chains. Compound (VIII) is the pentafluorophenyl analogue of (VII) and the molecules are again linked by $\mathrm{O}-\mathrm{H}$... O hydrogen bonds, but now forming cyclic $R_{6}^{6}(12)$ hexamers having exact $\overline{3}\left(S_{6}\right)$ symmetry (Ferguson et al., 1995).

\section{Synthesis and crystallization}

A sample of the title compound (I) was a gift from CAD Pharma, Bengaluru, India. Colourless blocks were grown by slow evaporation at room temperature of a solution in methanol, m.p. $478 \mathrm{~K}$.

\section{Refinement}

Crystal data, data collection and structure refinement details are summarized in Table 3. All $\mathrm{H}$ atoms were located in difference maps. The $\mathrm{H}$ atoms bonded to $\mathrm{C}$ atoms were then treated as riding atoms in geometrically idealized position with $\mathrm{C}-\mathrm{H}$ distances of $0.93 \AA$ (aromatic and heteroaromatic) or $0.98 \AA$ (aliphatic $\mathrm{CH}$ ) and with $U_{\text {iso }}(\mathrm{H})=1.2 U_{\text {eq }}(\mathrm{C})$. For the hydroxyl $\mathrm{H}$ atom $\mathrm{H} 1 A$, the atomic coordinates were refined with $U_{\text {iso }}(\mathrm{H})=1.5 U_{\text {eq }}(\mathrm{O})$, giving an $\mathrm{O}-\mathrm{H}$ distance of 0.84 (2) $\AA$. The analysis of variance reported a large value of $\mathrm{K}, 3.187$, for the group of 252 very weak reflections having $F_{\mathrm{c}} / F_{\mathrm{c}}(\max )$ in the range $0.000<F_{\mathrm{c}} / F_{\mathrm{c}}(\max )<0.005$.

\section{Acknowledgements}

$\mathrm{BN}$ thanks the UGC (India) for financial assistance. The X-ray data were collected at SAIF, IIT, Madras, India.

\section{References}

Bruker (2012). APEX2 and SAINT-Plus. Bruker AXS Inc., Madison, Wisconsin, USA.

Desiraju, G. R. \& Steiner, T. (1999). In The Weak Hydrogen Bond. Oxford University Press.

Ferguson, G., Carroll, C. D., Glidewell, C., Zakaria, C. M. \& Lough, A. J. (1995). Acta Cryst. B51, 367-377.

Ferguson, G., Gallagher, J. F., Glidewell, C., Low, J. N. \& Scrimgeour, S. N. (1992). Acta Cryst. C48, 1272-1275.

Ferguson, G., Gallagher, J. F., Glidewell, C. \& Zakaria, C. M. (1994). Acta Cryst. C50, 70-73.

Groom, C. R. \& Allen, F. H. (2014). Angew. Chem. Int. Ed. 53, 662671.

Imai, Y. N., Inoue, Y., Nakanishi, I. \& Kitaura, K. (2008). Protein Sci. 17, 1129-1137.

Kim, H. \& Kang, S. K. (2014). Acta Cryst. E70, o947.

Sheldrick, G. M. (2003). SADABS. University of Göttingen, Germany.

Sheldrick, G. M. (2008). Acta Cryst. A64, 112-122.

Sheldrick, G. M. (2015). Acta Cryst. C71, 3-8.

Shimada, H., Fujiki, S., Oginuma, M., Asakawa, M., Okawara, T., Kato, K., Yamamura, S., Akita, H., Hara, A. \& Imamura, Y. (2003). J. Mol. Catal. B Enzym. 23, 29-35.

Spek, A. L. (2009). Acta Cryst. D65, 148-155.

Tsang, M. Y., Di Salvo, F., Teixidor, F., Viñas, C., Planas, J. G., Choquesillo-Lazarte, D. \& Vanthuyne, N. (2015). Cryst. Growth Des. 15, 935-945. 


\section{supporting information}

Acta Cryst. (2016). E72, 69-72 [doi:10.1107/S2056989015023154]

\section{Crystal structure of $(R S)$-(4-chlorophenyl)(pyridin-2-yl)methanol}

\section{Badiadka Narayana, Balladka K. Sarojini, Hemmige S. Yathirajan, Ravindranath Rathore and Christopher Glidewell}

Computing details

Data collection: APEX2 (Bruker, 2012); cell refinement: SAINT-Plus (Bruker, 2012); data reduction: SAINT-Plus (Bruker, 2012); program(s) used to solve structure: SHELXS97 (Sheldrick, 2008); program(s) used to refine structure:

SHELXL2014 (Sheldrick, 2015); molecular graphics: PLATON (Spek, 2009); software used to prepare material for publication: SHELXL2014 (Sheldrick, 2015) and PLATON (Spek, 2009).

(RS)-(4-Chlorophenyl) (pyridin-2-yl)methanol

Crystal data

$\mathrm{C}_{12} \mathrm{H}_{10} \mathrm{CINO}$

$M_{r}=219.66$

Monoclinic, $P 2_{1} / c$

$a=8.4309(6) \AA$

$b=16.1488(11) \AA$

$c=8.6878(6) \AA$

$\beta=112.994(2)^{\circ}$

$V=1088.85(13) \AA^{3}$

$Z=4$

\section{Data collection}

\section{Bruker APEXII CCD}

diffractometer

Radiation source: fine-focus sealed tube

Graphite monochromator

$\varphi$ and $\omega$ scans

Absorption correction: multi-scan

(SADABS; Sheldrick, 2003)

$T_{\min }=0.719, T_{\max }=0.938$

\section{Refinement}

Refinement on $F^{2}$

Least-squares matrix: full

$R\left[F^{2}>2 \sigma\left(F^{2}\right)\right]=0.045$

$w R\left(F^{2}\right)=0.118$

$S=1.06$

2510 reflections

139 parameters

0 restraints
$F(000)=456$

$D_{\mathrm{x}}=1.340 \mathrm{Mg} \mathrm{m}^{-3}$

Mo $K \alpha$ radiation, $\lambda=0.71073 \AA$

Cell parameters from 2785 reflections

$\theta=2.5-28.6^{\circ}$

$\mu=0.32 \mathrm{~mm}^{-1}$

$T=295 \mathrm{~K}$

Block, colourless

$0.40 \times 0.30 \times 0.20 \mathrm{~mm}$

11481 measured reflections

2510 independent reflections

1860 reflections with $I>2 \sigma(I)$

$R_{\text {int }}=0.030$

$\theta_{\text {max }}=27.6^{\circ}, \theta_{\min }=2.5^{\circ}$

$h=-9 \rightarrow 10$

$k=-21 \rightarrow 15$

$l=-11 \rightarrow 11$

Hydrogen site location: mixed

$\mathrm{H}$ atoms treated by a mixture of independent and constrained refinement

$w=1 /\left[\sigma^{2}\left(F_{\mathrm{o}}^{2}\right)+(0.0513 P)^{2}+0.2352 P\right]$

where $P=\left(F_{\mathrm{o}}{ }^{2}+2 F_{\mathrm{c}}{ }^{2}\right) / 3$

$(\Delta / \sigma)_{\max }<0.001$

$\Delta \rho_{\max }=0.21 \mathrm{e} \AA^{-3}$

$\Delta \rho_{\min }=-0.39$ e $\AA^{-3}$ 


\section{Special details}

Geometry. All esds (except the esd in the dihedral angle between two 1.s. planes) are estimated using the full covariance matrix. The cell esds are taken into account individually in the estimation of esds in distances, angles and torsion angles; correlations between esds in cell parameters are only used when they are defined by crystal symmetry. An approximate (isotropic) treatment of cell esds is used for estimating esds involving l.s. planes.

Fractional atomic coordinates and isotropic or equivalent isotropic displacement parameters $\left(\AA^{2}\right)$

\begin{tabular}{lllll}
\hline & $x$ & $y$ & $z$ & $U_{\text {iso }} / U_{\text {eq }}$ \\
\hline C1 & $0.66425(18)$ & $0.19218(9)$ & $0.43123(18)$ & $0.0367(3)$ \\
H1 & 0.6340 & 0.2499 & 0.3976 & $0.044^{*}$ \\
O1 & $0.65282(15)$ & $0.17831(8)$ & $0.58744(14)$ & $0.0475(3)$ \\
H1A & $0.725(3)$ & $0.2105(14)$ & $0.655(3)$ & $0.071^{*}$ \\
C11 & $0.53625(18)$ & $0.13554(9)$ & $0.30436(19)$ & $0.0358(3)$ \\
C12 & $0.5356(2)$ & $0.05176(10)$ & $0.3383(2)$ & $0.0458(4)$ \\
H12 & 0.6128 & 0.0313 & 0.4400 & $0.055^{*}$ \\
C13 & $0.4232(2)$ & $-0.00168(11)$ & $0.2247(2)$ & $0.0533(5)$ \\
H13 & 0.4239 & -0.0578 & 0.2492 & $0.064^{*}$ \\
C14 & $0.3099(2)$ & $0.02875(12)$ & $0.0747(2)$ & $0.0548(5)$ \\
C114 & $0.16964(9)$ & $-0.03856(4)$ & $-0.07147(8)$ & $0.0922(3)$ \\
C15 & $0.3058(3)$ & $0.11173(14)$ & $0.0391(2)$ & $0.0668(6)$ \\
H15 & 0.2270 & 0.1321 & -0.0619 & $0.080^{*}$ \\
C16 & $0.4198(2)$ & $0.16462(11)$ & $0.1546(2)$ & $0.0538(5)$ \\
H16 & 0.4175 & 0.2208 & 0.1304 & $0.065^{*}$ \\
N21 & $0.88793(16)$ & $0.21277(8)$ & $0.32519(16)$ & $0.0414(3)$ \\
C22 & $0.84457(18)$ & $0.17470(9)$ & $0.43935(18)$ & $0.0339(3)$ \\
C23 & $0.9548(2)$ & $0.12092(11)$ & $0.5561(2)$ & $0.0461(4)$ \\
H23 & 0.9219 & 0.0959 & 0.6353 & $0.055^{*}$ \\
C24 & $1.1140(2)$ & $0.10498(12)$ & $0.5534(2)$ & $0.0537(5)$ \\
H24 & 1.1899 & 0.0688 & 0.6304 & $0.064^{*}$ \\
C25 & $1.1589(2)$ & $0.14314(12)$ & $0.4359(3)$ & $0.0570(5)$ \\
H25 & 1.2658 & 0.1335 & 0.4314 & $0.068^{*}$ \\
C26 & $1.0431(2)$ & $0.19599(11)$ & $0.3247(2)$ & $0.0523(4)$ \\
H26 & 1.0741 & 0.2216 & 0.2446 & $0.063^{*}$ \\
& & & & \\
\hline
\end{tabular}

Atomic displacement parameters $\left(\AA^{2}\right)$

\begin{tabular}{lllllll}
\hline & $U^{11}$ & $U^{22}$ & $U^{33}$ & $U^{12}$ & $U^{13}$ & $U^{23}$ \\
\hline C1 & $0.0359(8)$ & $0.0344(8)$ & $0.0421(8)$ & $0.0029(6)$ & $0.0178(7)$ & $0.0000(6)$ \\
O1 & $0.0520(7)$ & $0.0526(7)$ & $0.0455(7)$ & $-0.0078(6)$ & $0.0272(6)$ & $-0.0106(5)$ \\
C11 & $0.0323(7)$ & $0.0373(8)$ & $0.0411(8)$ & $0.0010(6)$ & $0.0177(6)$ & $-0.0001(6)$ \\
C12 & $0.0452(9)$ & $0.0401(9)$ & $0.0485(9)$ & $0.0034(7)$ & $0.0142(7)$ & $0.0020(7)$ \\
C13 & $0.0589(11)$ & $0.0406(9)$ & $0.0629(12)$ & $-0.0057(9)$ & $0.0265(10)$ & $-0.0055(8)$ \\
C14 & $0.0524(10)$ & $0.0617(12)$ & $0.0497(10)$ & $-0.0168(9)$ & $0.0194(8)$ & $-0.0134(9)$ \\
C114 & $0.0971(5)$ & $0.0942(5)$ & $0.0724(4)$ & $-0.0416(4)$ & $0.0190(3)$ & $-0.0316(3)$ \\
C15 & $0.0667(12)$ & $0.0685(13)$ & $0.0465(10)$ & $-0.0118(11)$ & $0.0019(9)$ & $0.0068(10)$ \\
C16 & $0.0554(10)$ & $0.0464(10)$ & $0.0502(10)$ & $-0.0044(8)$ & $0.0105(8)$ & $0.0095(8)$ \\
N21 & $0.0390(7)$ & $0.0417(7)$ & $0.0450(8)$ & $-0.0018(6)$ & $0.0182(6)$ & $0.0005(6)$
\end{tabular}




\begin{tabular}{|c|c|c|c|c|c|c|}
\hline $\mathrm{C} 22$ & $0.0341(7)$ & $0.0318(7)$ & $0.0351(8)$ & $-0.0016(6)$ & $0.0128(6)$ & $-0.0041(6)$ \\
\hline $\mathrm{C} 23$ & $0.0452(9)$ & $0.0492(9)$ & $0.0430(9)$ & $0.0064(8)$ & $0.0162(7)$ & $0.0031(8)$ \\
\hline $\mathrm{C} 24$ & $0.0418(9)$ & $0.0553(11)$ & $0.0563(11)$ & $0.0135(8)$ & $0.0108(8)$ & $-0.0005(9)$ \\
\hline $\mathrm{C} 25$ & $0.0357(8)$ & $0.0633(12)$ & $0.0748(13)$ & $0.0032(9)$ & $0.0245(9)$ & $-0.0093(10)$ \\
\hline $\mathrm{C} 26$ & $0.0459(9)$ & $0.0566(11)$ & $0.0637(11)$ & $-0.0050(9)$ & $0.0315(9)$ & $-0.0009(9)$ \\
\hline
\end{tabular}

Geometric parameters $\left(\hat{A},{ }^{\circ}\right)$

\begin{tabular}{|c|c|c|c|}
\hline $\mathrm{C} 1-\mathrm{O} 1$ & $1.4154(18)$ & $\mathrm{C} 15-\mathrm{C} 16$ & $1.381(3)$ \\
\hline $\mathrm{C} 1-\mathrm{C} 11$ & $1.512(2)$ & $\mathrm{C} 15-\mathrm{H} 15$ & 0.9300 \\
\hline $\mathrm{C} 1-\mathrm{C} 22$ & $1.5206(19)$ & $\mathrm{C} 16-\mathrm{H} 16$ & 0.9300 \\
\hline $\mathrm{C} 1-\mathrm{H} 1$ & 0.9800 & $\mathrm{~N} 21-\mathrm{C} 22$ & $1.3335(19)$ \\
\hline $\mathrm{O} 1-\mathrm{H} 1 \mathrm{~A}$ & $0.84(2)$ & $\mathrm{N} 21-\mathrm{C} 26$ & $1.338(2)$ \\
\hline $\mathrm{C} 11-\mathrm{C} 16$ & $1.371(2)$ & $\mathrm{C} 22-\mathrm{C} 23$ & $1.382(2)$ \\
\hline $\mathrm{C} 11-\mathrm{C} 12$ & $1.385(2)$ & $\mathrm{C} 23-\mathrm{C} 24$ & $1.376(2)$ \\
\hline $\mathrm{C} 12-\mathrm{C} 13$ & $1.373(2)$ & $\mathrm{C} 23-\mathrm{H} 23$ & 0.9300 \\
\hline $\mathrm{C} 12-\mathrm{H} 12$ & 0.9300 & $\mathrm{C} 24-\mathrm{C} 25$ & $1.367(3)$ \\
\hline $\mathrm{C} 13-\mathrm{C} 14$ & $1.371(3)$ & $\mathrm{C} 24-\mathrm{H} 24$ & 0.9300 \\
\hline $\mathrm{C} 13-\mathrm{H} 13$ & 0.9300 & $\mathrm{C} 25-\mathrm{C} 26$ & $1.370(3)$ \\
\hline $\mathrm{C} 14-\mathrm{C} 15$ & $1.373(3)$ & $\mathrm{C} 25-\mathrm{H} 25$ & 0.9300 \\
\hline $\mathrm{C} 14-\mathrm{Cl14}$ & $1.7376(18)$ & $\mathrm{C} 26-\mathrm{H} 26$ & 0.9300 \\
\hline $\mathrm{O} 1-\mathrm{C} 1-\mathrm{C} 11$ & $107.86(12)$ & $\mathrm{C} 16-\mathrm{C} 15-\mathrm{H} 15$ & 120.3 \\
\hline $\mathrm{O} 1-\mathrm{C} 1-\mathrm{C} 22$ & $111.56(12)$ & $\mathrm{C} 11-\mathrm{C} 16-\mathrm{C} 15$ & $121.00(17)$ \\
\hline $\mathrm{C} 11-\mathrm{C} 1-\mathrm{C} 22$ & $109.72(11)$ & $\mathrm{C} 11-\mathrm{C} 16-\mathrm{H} 16$ & 119.5 \\
\hline $\mathrm{O} 1-\mathrm{C} 1-\mathrm{H} 1$ & 109.2 & $\mathrm{C} 15-\mathrm{C} 16-\mathrm{H} 16$ & 119.5 \\
\hline $\mathrm{C} 11-\mathrm{C} 1-\mathrm{H} 1$ & 109.2 & $\mathrm{C} 22-\mathrm{N} 21-\mathrm{C} 26$ & $117.48(14)$ \\
\hline $\mathrm{C} 22-\mathrm{C} 1-\mathrm{H} 1$ & 109.2 & $\mathrm{~N} 21-\mathrm{C} 22-\mathrm{C} 23$ & $122.29(13)$ \\
\hline $\mathrm{C} 1-\mathrm{O} 1-\mathrm{H} 1 \mathrm{~A}$ & $105.9(15)$ & $\mathrm{N} 21-\mathrm{C} 22-\mathrm{C} 1$ & $116.07(13)$ \\
\hline $\mathrm{C} 16-\mathrm{C} 11-\mathrm{C} 12$ & $118.40(15)$ & $\mathrm{C} 23-\mathrm{C} 22-\mathrm{C} 1$ & $121.63(13)$ \\
\hline $\mathrm{C} 16-\mathrm{C} 11-\mathrm{C} 1$ & $121.82(14)$ & $\mathrm{C} 24-\mathrm{C} 23-\mathrm{C} 22$ & $119.09(16)$ \\
\hline $\mathrm{C} 12-\mathrm{C} 11-\mathrm{C} 1$ & $119.78(14)$ & $\mathrm{C} 24-\mathrm{C} 23-\mathrm{H} 23$ & 120.5 \\
\hline $\mathrm{C} 13-\mathrm{C} 12-\mathrm{C} 11$ & $121.32(16)$ & $\mathrm{C} 22-\mathrm{C} 23-\mathrm{H} 23$ & 120.5 \\
\hline $\mathrm{C} 13-\mathrm{C} 12-\mathrm{H} 12$ & 119.3 & $\mathrm{C} 25-\mathrm{C} 24-\mathrm{C} 23$ & $119.03(17)$ \\
\hline $\mathrm{C} 11-\mathrm{C} 12-\mathrm{H} 12$ & 119.3 & $\mathrm{C} 25-\mathrm{C} 24-\mathrm{H} 24$ & 120.5 \\
\hline $\mathrm{C} 14-\mathrm{C} 13-\mathrm{C} 12$ & $119.13(17)$ & $\mathrm{C} 23-\mathrm{C} 24-\mathrm{H} 24$ & 120.5 \\
\hline $\mathrm{C} 14-\mathrm{C} 13-\mathrm{H} 13$ & 120.4 & $\mathrm{C} 24-\mathrm{C} 25-\mathrm{C} 26$ & $118.56(15)$ \\
\hline $\mathrm{C} 12-\mathrm{C} 13-\mathrm{H} 13$ & 120.4 & $\mathrm{C} 24-\mathrm{C} 25-\mathrm{H} 25$ & 120.7 \\
\hline $\mathrm{C} 13-\mathrm{C} 14-\mathrm{C} 15$ & $120.73(17)$ & $\mathrm{C} 26-\mathrm{C} 25-\mathrm{H} 25$ & 120.7 \\
\hline $\mathrm{C} 13-\mathrm{C} 14-\mathrm{Cl14}$ & $119.54(15)$ & $\mathrm{N} 21-\mathrm{C} 26-\mathrm{C} 25$ & $123.55(16)$ \\
\hline $\mathrm{C} 15-\mathrm{C} 14-\mathrm{Cl14}$ & $119.73(15)$ & $\mathrm{N} 21-\mathrm{C} 26-\mathrm{H} 26$ & 118.2 \\
\hline $\mathrm{C} 14-\mathrm{C} 15-\mathrm{C} 16$ & $119.40(18)$ & $\mathrm{C} 25-\mathrm{C} 26-\mathrm{H} 26$ & 118.2 \\
\hline $\mathrm{C} 14-\mathrm{C} 15-\mathrm{H} 15$ & 120.3 & & \\
\hline $\mathrm{O} 1-\mathrm{C} 1-\mathrm{C} 11-\mathrm{C} 16$ & $129.16(15)$ & $\mathrm{C} 26-\mathrm{N} 21-\mathrm{C} 22-\mathrm{C} 23$ & $1.2(2)$ \\
\hline $\mathrm{C} 22-\mathrm{C} 1-\mathrm{C} 11-\mathrm{C} 16$ & $-109.13(16)$ & $\mathrm{C} 26-\mathrm{N} 21-\mathrm{C} 22-\mathrm{C} 1$ & $-177.31(14)$ \\
\hline $\mathrm{O} 1-\mathrm{C} 1-\mathrm{C} 11-\mathrm{C} 12$ & $-51.14(17)$ & $\mathrm{O} 1-\mathrm{C} 1-\mathrm{C} 22-\mathrm{N} 21$ & $-156.41(13)$ \\
\hline $\mathrm{C} 22-\mathrm{C} 1-\mathrm{C} 11-\mathrm{C} 12$ & $70.57(17)$ & $\mathrm{C} 11-\mathrm{C} 1-\mathrm{C} 22-\mathrm{N} 21$ & $84.12(16)$ \\
\hline
\end{tabular}




$\begin{array}{llll}\mathrm{C} 16-\mathrm{C} 11-\mathrm{C} 12-\mathrm{C} 13 & 0.9(2) & \mathrm{O} 1-\mathrm{C} 1-\mathrm{C} 22-\mathrm{C} 23 & 25.1(2) \\ \mathrm{C} 1-\mathrm{C} 11-\mathrm{C} 12-\mathrm{C} 13 & -178.84(14) & \mathrm{C} 11-\mathrm{C} 1-\mathrm{C} 22-\mathrm{C} 23 & -94.42(16) \\ \mathrm{C} 11-\mathrm{C} 12-\mathrm{C} 13-\mathrm{C} 14 & 0.2(3) & \mathrm{N} 21-\mathrm{C} 22-\mathrm{C} 23-\mathrm{C} 24 & -1.0(2) \\ \mathrm{C} 12-\mathrm{C} 13-\mathrm{C} 14-\mathrm{C} 15 & -1.3(3) & \mathrm{C} 1-\mathrm{C} 22-\mathrm{C} 23-\mathrm{C} 24 & 177.48(15) \\ \mathrm{C} 12-\mathrm{C} 13-\mathrm{C} 14-\mathrm{C} 114 & 178.90(13) & \mathrm{C} 22-\mathrm{C} 23-\mathrm{C} 24-\mathrm{C} 25 & 0.3(3) \\ \mathrm{C} 13-\mathrm{C} 14-\mathrm{C} 15-\mathrm{C} 16 & 1.4(3) & \mathrm{C} 23-\mathrm{C} 24-\mathrm{C} 25-\mathrm{C} 26 & 0.0(3) \\ \mathrm{C} 114-\mathrm{C} 14-\mathrm{C} 15-\mathrm{C} 16 & -178.83(15) & \mathrm{C} 22-\mathrm{N} 21-\mathrm{C} 26-\mathrm{C} 25 & -0.9(3) \\ \mathrm{C} 12-\mathrm{C} 11-\mathrm{C} 16-\mathrm{C} 15 & -0.8(3) & \mathrm{C} 24-\mathrm{C} 25-\mathrm{C} 26-\mathrm{N} 21 & 0.3(3) \\ \mathrm{C} 1-\mathrm{C} 11-\mathrm{C} 16-\mathrm{C} 15 & 178.90(16) & \mathrm{C} 11-\mathrm{C} 1-\mathrm{O} 1-\mathrm{H} 1 \mathrm{~A} & -180.0(17) \\ \mathrm{C} 14-\mathrm{C} 15-\mathrm{C} 16-\mathrm{C} 11 & -0.3(3) & & \end{array}$

Hydrogen-bond geometry $\left(\AA,{ }^{\circ}\right)$

\begin{tabular}{lllll}
\hline$D-\mathrm{H} \cdots A$ & $D-\mathrm{H}$ & $\mathrm{H} \cdots A$ & $D \cdots A$ & $D-\mathrm{H} \cdots A$ \\
\hline $\mathrm{O} 1-\mathrm{H} 1 A \cdots \mathrm{N} 21^{\mathrm{i}}$ & $0.84(2)$ & $2.01(2)$ & $2.8444(18)$ & $176(2)$
\end{tabular}

Symmetry code: (i) $x,-y+1 / 2, z+1 / 2$. 